\title{
Clinical profile of Alzheimer's patients: A multihospital based study done in India
}

\author{
Nitin Joseph, Sarthak Sharma, Madhur Khullar, Sreemanth Chede, Ann Cheruvillil Joy, \\ Gauri Thukral \\ Kasturba Medical College, Manipal Academy of Higher Education, Mangalore, India
}

\begin{abstract}
Objectives. To find out the risk factors, clinical features, co-morbid conditions and management practices among $A D$ patients.

Materials and methods. This study was conducted at three major tertiary care hospitals in Mangalore city of south India. Medical records of confirmed cases of AD over the recent 10 years were analyzed. A semi-structured proforma was used for data collection.

Outcomes. Mean age of the 55 AD patients was $81.1 \pm 7.6$ years. The mean age at diagnosis was $75.95 \pm 7.95$ years The age at the onset of $A D$ in $14(25.5 \%)$ patients was from 76 to 80 years. Early onset $A D$ ( $\leq 65$ years) was reported among $9(16.4 \%)$ patients. $35(63.6 \%)$ patients were males and $37(67.3 \%)$ were from urban area. Family history of AD was present among $2(3.6 \%)$ patients. History of depression and traumatic brain injury was present among 5 $(9.1 \%)$ and $4(7.3 \%)$ patients respectively. History of consumption of alcohol and smoking was present in $9(16.4 \%)$ and $7(12.7 \%)$ patients respectively. Clinical features such as disorientation and recent memory loss was present among $39(70.9 \%)$ and $38(69.1 \%)$ patients respectively. Donepezil was used for the management of AD in $44(80 \%)$ of patients. Non-pharmacological methods like physiotherapy were recommended in 21 (38.2\%) AD patients.

Conclusions. Considering the varied problems faced by the Alzheimer's affected patients in terms of co-morbidities and clinical presentation, a multidisciplinary approach for the management of $A D$ is suggested. More non-pharmacologic methods need to be introduced at this setting.
\end{abstract}

Keywords: Alzheimer's disease, risk factors, clinical features, management, hospital based study

\section{INTRODUCTION}

Alzheimer's disease (AD) is an age related degenerative disease of the brain. It accounts for as much as $60-80 \%$ of disorders associated with dementia in the older people [1]. The Global Burden of Disease Study report stated that the prevalence of $\mathrm{AD}$ and other dementias was 2933814 (Uncertainty Interval 2466582-3493076), in India, for the year 2016 [2].

The global burden of AD is on the rise and is estimated to increase by about $35 \%$ by the end of 2025 [3]. Risk of AD has declined in the higher-income western countries [4,5], however, it is substantial in the low and middle income countries [6].
It is also the most common cause of disability and morbidity among the older people [1].

Genetic and environmental factors are known to cause AD [7]. Reports of increasing prevalence of $\mathrm{AD}$ makes it essential in identifying more risk factors of the same. This will help in framing preventive strategies for patients at risk. Experts believe that there are multiple risk factors for $\mathrm{AD}$ as also seen in other chronic diseases [1]. Thus intervention on multiple risk factors simultaneously would increase the chances of risk reduction than addressing a single risk factor alone.

Information about its etio-pathogenesis and progression is very limited [8]. Hence it was also important to study its clinical presentation in the cur- 
rent settings. The benefits of early investigation and diagnosis of $\mathrm{AD}$ includes timely initiation of medications and provision of non-pharmacological support measures to these patients. Management of its common comorbid conditions would further help in improving the quality of life of the affected. This study was therefore done to find out the risk factors, clinical features, co-morbid conditions and management practices among AD patients in various tertiary care hospitals in a coastal city of south India.

\section{MATERIALS AND METHODS}

This record based retrospective study was conducted in the month of May 2018. Permission to conduct this study was obtained from the Institutional Ethics Committee (IEC). The study was thus performed in compliance with the ethical principles of the assigned institutional board.

Following this, the study was done at two tertiary care private hospitals and a tertiary care government hospital, affiliated to a private medical college in Mangalore. The medical superintendents of the respective hospitals gave permission to obtain the information from the medical record section.

Hospital records of patients confirmed with $\mathrm{AD}$ over the recent 10 years from January 2008 to May 2018 were analyzed by the investigators. Relevant data were recorded in a semi-structured proforma. Content validation of the proforma was done by subject experts.

Data entry and analysis were done using SPSS Inc., Chicago, IL, USA) version 17.0. Data were presented in categories and percentages. Fisher's exact test was used to test association. $\mathrm{p}$ value $\leq$ 0.05 was considered as statistically significant association.

\section{RESULTS}

Of the 55 patients with Alzheimer's disease, $19(34.5 \%)$ were from the government hospital and the rest $36(65.5 \%)$ were from the two private hospitals. The mean age of the patients was $81.1 \pm 7.6$ years. The mean age at diagnosis of the patients was $75.95 \pm 7.95$ years.

The age at the onset of $\mathrm{AD}$ in $14(25.5 \%)$ patients was from 76 to 80 years. Early onset AD $(\leq 65$ years) was reported among 9(16.4\%) patients. Among the AD patients, 35(63.6\%) were males and $37(67.3 \%)$ were from urban area (Table 1$)$.

TABLE 1. Socio demographic distribution of Alzheimer's disease patients $(n=55)$

\begin{tabular}{|c|c|c|}
\hline Characteristics & Number & Percentage \\
\hline \multicolumn{3}{|c|}{ Age group (years) } \\
\hline $65-70$ & 7 & 12.7 \\
\hline 71-75 & 6 & 10.9 \\
\hline $76-80$ & 13 & 23.7 \\
\hline $81-85$ & 12 & 21.8 \\
\hline $86-90$ & 10 & 18.2 \\
\hline $91-95$ & 7 & 12.7 \\
\hline \multicolumn{3}{|c|}{ Age at diagnosis (years) } \\
\hline $61-65$ & 9 & 16.4 \\
\hline $66-70$ & 5 & 9.1 \\
\hline $71-75$ & 10 & 18.2 \\
\hline $76-80$ & 14 & 25.4 \\
\hline $81-85$ & 10 & 18.2 \\
\hline $86-90$ & 7 & 12.7 \\
\hline \multicolumn{3}{|l|}{ Gender } \\
\hline Males & 35 & 63.6 \\
\hline Females & 20 & 36.4 \\
\hline \multicolumn{3}{|l|}{ Marital status } \\
\hline Married & 55 & 100.0 \\
\hline \multicolumn{3}{|c|}{ Occupational status $(n=25)$} \\
\hline Retired & 23 & 92.0 \\
\hline Working & 2 & 8.0 \\
\hline \multicolumn{3}{|l|}{ Place } \\
\hline Urban & 37 & 67.3 \\
\hline Rural & 18 & 32.7 \\
\hline
\end{tabular}

TABLE 2. Distribution of risk factors of Alzheimer's disease among patients $(n=55)$

\begin{tabular}{|l|c|c|}
\hline Risk factors & Number & Percentage \\
\hline History of alcohol consumption & 9 & 16.4 \\
\hline History of smoking & 7 & 12.7 \\
\hline Depression as a co-morbidity & 5 & 9.1 \\
\hline History of traumatic brain injury & 4 & 7.3 \\
\hline Meningitis as a co-morbidity & 2 & 3.6 \\
\hline $\begin{array}{l}\text { Presence of family history of } \\
\text { Alzheimer's disease }\end{array}$ & 2 & 3.6 \\
\hline Presence of dementia & 14 & 25.4 \\
\hline Type of dementia & & \\
\hline Dementia associated with Parkinsonism & 10 & 18.2 \\
\hline Vascular dementia & 5 & 9.1 \\
\hline Dementia associated with Lewi bodies & 1 & 1.8 \\
\hline Vitamin deficiency & 1 & 1.8 \\
\hline
\end{tabular}

History of depression and of traumatic brain injury (TBI) was present among 5(9.1\%) and 4(7.3\%) patients respectively (Table 2 ).

Family history of AD was present in two patients of which, one presented with early onset AD $(\mathrm{p}=0.303)$. 
TABLE 3. Distribution of co-morbidities in Alzheimer's disease among patients $(n=55)$

\begin{tabular}{|c|c|c|}
\hline Co-morbidities & Number & Percentage \\
\hline Hypertension & 33 & 60.0 \\
\hline Diabetes mellitus & 16 & 29.1 \\
\hline Benign prostatic hypertrophy & 12 & 21.8 \\
\hline Urinary tract infection & 11 & 20.0 \\
\hline Ischaemic heart disease & 10 & 18.2 \\
\hline Parkinsonism & 10 & 18.2 \\
\hline Pneumonia & 9 & 16.4 \\
\hline COPD & 6 & 10.9 \\
\hline Osteo arthritis & 5 & 9.1 \\
\hline Chronic kidney disease & 4 & 7.3 \\
\hline Dysphagia & 4 & 7.3 \\
\hline Pressure sores & 4 & 7.3 \\
\hline Respiratory failure & 4 & 7.3 \\
\hline Sepsis & 3 & 5.4 \\
\hline Chronic liver disease & 3 & 5.4 \\
\hline Cerebro vascular accidents & 2 & 3.6 \\
\hline Gastroenteritis & 2 & 3.6 \\
\hline Tinnitus & 2 & 3.6 \\
\hline Others $^{\dagger}$ & 9 & 16.4 \\
\hline \multicolumn{3}{|l|}{ No. of co-morbidities } \\
\hline $\mathrm{Nil}$ & 5 & 9.1 \\
\hline $1-2$ & 29 & 52.7 \\
\hline $3-4$ & 15 & 27.3 \\
\hline $5-7$ & 6 & 10.9 \\
\hline
\end{tabular}

${ }^{+}$Hypercholesterolemia 1, dyslipidaemia 1, arrhythmias 1, asthma 1, aortic sclerosis 1 , hepatocellular carcinoma 1 , Fournier's gangrene 1 , Mallory Weiss syndrome 1, hypothyroidism 1

Fifty $(90.9 \%)$ patients had at least one co-morbidity. The most common co-morbidity among patients was hypertension [33(60\%)] (Table 3).

TABLE 4. Clinical presentation in Alzheimer's disease among patients $(n=55)$

\begin{tabular}{|l|c|c|}
\hline Clinical features & Number & Percentage \\
\hline Disorientation & 39 & 70.9 \\
\hline Recent memory loss & 38 & 69.1 \\
\hline Disturbed sleep & 20 & 36.4 \\
\hline Urinary incontinence & 19 & 34.5 \\
\hline Cognitive impairment & 15 & 27.3 \\
\hline Increased agitation & 14 & 25.4 \\
\hline Imbalance in gait & 14 & 25.4 \\
\hline Aphasia & 13 & 23.6 \\
\hline Difficulty with routine activities & 12 & 21.8 \\
\hline Long term memory loss & 10 & 18.2 \\
\hline History of fall & 10 & 18.2 \\
\hline Seizures & 8 & 14.5 \\
\hline Bowel incontinence & 7 & 12.7 \\
\hline Slowness in response & 6 & 10.9 \\
\hline Forgetting dates and events & 6 & 10.9 \\
\hline Vision problems & 5 & 9.1 \\
\hline Apraxia & 5 & 9.1 \\
\hline $\begin{array}{l}\text { Putting things in unusual places } \\
\text { or losing them }\end{array}$ & 4 & 7.3 \\
\hline
\end{tabular}

\begin{tabular}{|l|c|c|}
\hline Clinical features & Number & Percentage \\
\hline Paranoia & 4 & 7.3 \\
\hline Syncope & 4 & 7.3 \\
\hline Agnosia & 3 & 5.4 \\
\hline Asking for same information & 3 & 5.4 \\
\hline Others $^{\ddagger}$ & 11 & 20.0 \\
\hline
\end{tabular}

${ }^{\ddagger}$ Getting lost in familiar places 2, Psychosis 2, Drowsiness 2, Dyscalculia 1, Irritability 1, Flapping tremors 1, Hallucination 1, Delirium 1

Disorientation and recent memory loss was present among 39(70.9\%) and 38(69.1\%) patients respectively (Table 4).

The various investigations done among patients included CT scan in $13(23.6 \%)$ and MRI scan in $7(12.7 \%)$.

TABLE 5. Medications prescribed for Alzheimer's disease patients $(n=55)$

\begin{tabular}{|l|c|c|}
\hline Medications & Number & Percentage \\
\hline Donepezil & 44 & 80.0 \\
\hline Memantine & 26 & 47.3 \\
\hline Quetiapine & 17 & 30.9 \\
\hline Vitamin supplements & 9 & 16.4 \\
\hline Zolpidem & 5 & 9.1 \\
\hline Levodopa with Carbidopa & 5 & 9.1 \\
\hline Piracetam & 3 & 5.4 \\
\hline Lorazepam & 2 & 3.6 \\
\hline Olanzapine & 1 & 1.8 \\
\hline
\end{tabular}

Donepezil was used for management in $44(80 \%)$ AD patients (Table 5).

Non-pharmacological management advised to patients comprised of physiotherapy in $21(38.2 \%)$, diet rich in polyunsaturated fatty acids (PUFA) in 6 $(10.9 \%)$, yoga and exercises in 2 patients each.

\section{DISCUSSION}

In the present study, the age at the onset of majority of the AD patients was from 76 to 80 years. The proportion of patients with the age at onset $\geq$ 85 years and $\geq 75$ years in the present study was $12.7 \%$ and $56.4 \%$ respectively. This was much lesser than the findings of an American study where, $32 \%$ AD patients were $\geq 85$ years and $82 \%$ were $\geq$ 75 years of age [3]. Another Indian study observed that, majority $(35.4 \%)$ of the patients had the onset of $\mathrm{AD}$ in the age group 60-64 years [9]. The reason for the delayed onset of $\mathrm{AD}$ in the US population could because of higher frequency of the APOE $\varepsilon 4$ allele among Americans compared to Indians [10]. The APOE $\varepsilon 4$ allele is the major risk-factor gene 
for $\mathrm{AD}$, and it results in the onset of $\mathrm{AD}$ at a later age [11].

This study also observed that the proportion of early onset $\mathrm{AD}$ patients were $16.4 \%$. This was more than the expected proportion of 5 to $10 \%$ as reported by Grossberg et al. [12]. Early-onset AD patients usually have history of familial AD. The inheritance pattern being that of autosomal dominant variety [12]. Abnormal genes located on chromosomes 21,14 , and 1, account for majority of patients with early-onset AD [13]. Mutations involve the gene for presenilin 1 and 2 proteins and amyloid precursor protein [1]. For the patients with early-onset familial $\mathrm{AD}$, referral services for them as well as their family members to a genetic counsellor is recommended [12]. This will help in identifying others in the family pedigree at greater risk of developing AD. However the association between family history of $\mathrm{AD}$ and early onset $\mathrm{AD}$ could not be established in the present study.

In this study, almost two-third of patients were males. On the contrary, in the USA based study [3], almost two-third of the patients with AD were females and a Taiwanese study [14] reported female gender as a risk factor for AD.

More than two-third of the AD patients in this study were from urban areas. The Taiwanese study reported urbanization status as an independent risk factor for AD [14]. Similarly, a study done in USA reported that the adjusted prevalence ratios of $\mathrm{AD}$ and related disorders remained $11 \%$ lower in rural compared to the urban counties [15]. Reason for greater number of $\mathrm{AD}$ patients in urban areas of the world could be due to greater prevalence of NCDs in these areas as a consequence of faulty lifestyle habits. The NCDs affect the vascular system and increases the risk of dementias including AD [16].

Family history of AD was reported to be positive among 3.6\% patients in this study. This was supported by a previous observation where familial $\mathrm{AD}$ accounted for $<5 \%$ of all patients with $\mathrm{AD}$ [17]. In patients with familial $A D$, chances of inheriting the disease-causing gene is increased in their children, thereby increasing their risk of developing $\mathrm{AD}$ [17]. The first degree relatives of $\mathrm{AD}$ patients were found to have three times greater risk than unrelated people [7].

Co-morbidities like hypertension, DM and ischemic heart disease were present among number of patients in this study. These diseases induces dysfunction of the blood vessels which result in chronic brain hypo perfusion leading to metabolic stress in neurons [18]. The state of cerebrovascular damage and long standing hypo perfusion of the brain is prodromal to AD [19]. Previous studies have also supported DM [7,14,17] and hypertension $[7,14]$ as risk factors of AD.

History of smoking and alcoholism was present among $12.7 \%$ and $16.4 \%$ patients respectively in this study. Smoking as a risk factor for AD has been supported in previous literature [17]. It was also observed that the risk of developing $\mathrm{AD}$ reduced among ex-smokers [17]. This meant that quitting smoking would result in reduction in the risk of dementia [17]. The same study also found that excessive alcohol consumers had the highest risk of developing $\mathrm{AD}$ [17].

Other risk factors of dementia reported were stroke [14], atrial fibrillation [7] and high cholesterol levels [7,17]. Factors that increase the risk of cardiovascular diseases were hence found to increase the risk of dementia. Management of cardiovascular risk factors such as smoking would be therefore beneficial in reducing the risk of decline in cognition and of dementia in the future [7].

Poor sleep quality and sleep disorders [20] and low cognitive activity [7] were other non-cardiovascular risk factors of $\mathrm{AD}$ identified in previous studies.

Depression was an important co-morbidity in AD reported in previous studies [7,21] as also observed among 9.1\% patients in the present study. Paranoia was observed here to be present among $7.3 \%$ AD patients. Emotional changes are common in $\mathrm{AD}$ and previous studies have reported major depression, anxiety, apathy and delusions in 24 to $32 \%, 17$ to $27 \%$, upto $41 \%$ and $23 \%$ AD patients respectively [21]. It has been estimated that $50 \%$ of the global AD patients may be the result of seven key modifiable risk factors namely, depression, hypertension, DM, obesity, cognitive and physical inactivity along with behaviours like smoking [17]. The role of a clinical psychologist is therefore vital to provide advices in behavioural approaches and to address depression in AD management [12].

History of TBI was present in $7.3 \%$ patients. It is a known risk factor for $\mathrm{AD}$ [7]. Repeated head injuries sustained in boxing, football and other con- 
tact sports, results in chronic traumatic encephalopathy (CTE), behavioural or cognitive dysfunction and increases the risk of dementia [1]. It happens because of over-expression of the $\beta$-amyloid precursor protein as a consequence of head injury which further leads to the accumulation of $\beta$-amyloid deposits in the brain. This pathogenesis is similar to the neural changes in AD [22]. Another theory suggests, head injury results in altered brain vasculature, worsens hippocampal degeneration and thereby accelerates the progression of AD pathology [23]. Burns et al. reported a positive relationship between head injury and AD and other neurodegenerative diseases [7].

In the present study, $18.2 \%$ patients had parkinsonism. This along with other medical conditions like chronic kidney disease, multiple sclerosis, and HIV increase the risk of developing dementia [17].

The other common co-morbidity of AD observed in this study was benign prostatic hypertrophy which was seen among $21.8 \%$ patients. This can lead to urinary tract infections which was observed among $20 \%$ patients in this study. It is known to result in delirium which is another significant problem in $\mathrm{AD}$ [21].

The present study observed that $16.4 \%$ AD patients had pneumonia. This usually happens in severe AD patients who suffer with difficulty in ambulation and become vulnerable to infections such as pneumonia [1]. In older patients with $\mathrm{AD}$ and other dementias, pneumonia was found to be the most common cause of death [24]. Hence this condition needs to be diagnosed early among AD patients and managed appropriately.

In this study, recent memory loss was reported by $69.1 \%$ of patients. This has been reported by Burns et al. to be the most common presenting symptoms in AD patients [7]. This along with language difficulties and executive dysfunction are the other symptoms related to cognitive dysfunction in $\mathrm{AD}$ [7].

Disorientation was reported among $70.9 \% \mathrm{AD}$ patients and it happened to be the most common presenting symptom in this study. Agitation was reported by $25.4 \%$ patients and hallucination by one patient. These symptoms along with delusions and depression constitutes non-cognitive symptoms. Apart from the cognitive and non-cognitive symptoms, there are symptoms related to difficulties with performing activities of daily living [7]. This was reported by $21.8 \%$ participants in the present study.

Several co-morbidities are common in AD as a consequence of the dementing process involving this disease. In this study, $90.9 \%$ of AD patients had at least one co-morbidity and $38.2 \%$ of them had atleast three co-morbidities. Leon et al. reported that $93 \%$ of $\mathrm{AD}$ patients had atleast one co-morbidity while more than $60 \%$ of them had 3 or more comorbid conditions [25].

It is important to diagnose $\mathrm{AD}$ early and start treatment as soon as possible. Without treatment, symptoms like memory loss, anxiety and depression in the initial stages of AD lasts for 2 to 3 years. In the moderate stage which follows, delusions, visual hallucinations, reversal of sleep patterns and other neuropsychiatric manifestations emerge, while the initial stage symptoms disappear. Finally, comes the severe stage which is characterized by motor rigidity and cognitive decline [12].

The drugs used in treatment of AD do not stop neuronal damage [1]. It only increases the levels of neurotransmitters temporarily and gives symptomatic improvement to AD patients [1]. Acetyl cholinesterase inhibitors (AChEIs) are the main drugs used in the treatment for $\mathrm{AD}$ [26]. It has been shown to have symptom modifying effect and is also well tolerated in several AD patients [7]. In this study too, $80 \%$ of patients were managed using Donepezil. It is the most commonly prescribed AChEI [27]. This drug has been approved for the treatment of early/ mild to moderate type of AD [12,27]. No other AChEIs like rivastigmine or galantamine were prescribed to AD patients in this study. Donepezil has the advantage over the other drugs in the family due to its once-a-day dosing [12] Combination of AChEIs for AD management was also not observed in the present study, which was again as per recommendations [12]. Early initiation of AChEIs in mild to moderate AD patients, helps in delaying the onset of neuropsychiatric symptoms.

Recent trials however found that Donepezil was ineffective in improving behavioural problems [ ${ }^{28]}$ As AChEI do not alter the underlying disease process, its long-lasting effect is also limited [27].

The next common drug used in this study for treatment in close to half of the AD patients was Memantine. This is a glutamatergic partial antago- 
nist used to manage moderate to severe $\mathrm{AD}[7,12]$. Both Donepezil and Memantine have been reported to cause improvement in activities of daily living among the AD affected patients [26].

The third most common drug used for management was Quetiapine, used in about one-third of the AD patients. This second generation antipsychotic drug along with Olanzapine, although reduces agitated behaviour, is known for side effects like higher risk of stroke and impairment of cognition [7].

One of the side effect of AChEIs like Donepezil is that it can precipitate or exacerbate urinary incontinence. This may be one of the reason behind more than a third of AD patients having urinary incontinence in this study. These patients need to be trained by scheduled toileting, prompted voiding and behaviour modification to improve their quality of life [12].

A short course of benzodiazepines may be used to alleviate moderate to severe levels of anxiety in $\mathrm{AD}$ patients. Lorazepam has a short half-life and is therefore recommended. In this study, Lorazepam was prescribed to two patients. Prolonged usage of benzodiazepines is not recommended, due to decline in its efficacy over time and due its side effects like sedation, loss of coordination and impairment of cognitive functioning [29]. Antipsychotics drugs are recommended as they perform better than benzodiazepines in reducing agitation and in control of moderate to severe behavioural disturbances in $\mathrm{AD}$ patients [12].

Vitamin supplements were prescribed in $16.4 \%$ AD patients. Vitamins B6, B9 and B12 are known to delay cognitive ageing [30]. When vitamin B9 or B12 levels are deficient in the body, the homocysteine level increases. This increases the risk of neuronal death and also contributes to accumulation of tau protein and amyloid. All these lead to increased susceptibility to develop AD. Rise in homocysteine level also predisposes to cardiovascular diseases [30]. In addition, vitamins like $\mathrm{C}$ and $\mathrm{E}$ have antioxidant properties which limit damage by free radicals, prevents oxidative damage and neurodegeneration, which would have otherwise resulted in dementia [30]. The incidence of cognitive decline and dementia has been found to decline with increasing levels of vitamin $\mathrm{E}$ in the body [31]. Vita$\min \mathrm{E}$ also slows the progression of functional and cognitive decline in $\mathrm{AD}$ [32].
Diet rich in PUFA was advised to six patients in the present study. Dietary omega-3 PUFA is essential for neuronal growth and influence synapse formations. It is thus beneficial in the inflammatory, vascular and amyloid pathways of dementia [30]. In addition, consuming a healthy diet with minimal saturated fatty acids would benefit the cardio vascular system and minimize the risk of $\mathrm{AD}$ and dementia. Apart from dietary modifications, the other non-pharmacological advises given to $\mathrm{AD}$ patients in the present study were physiotherapy, exercise and yoga. Previously done systematic review has found that exercises benefit AD patients by improving cognitive functions and by slowing down the progression of cognitive decline [33]. Ongoing trials support the value of yoga and physical activity [34]. In addition, light exercises such as walking may decrease behavioural problems [12]. Exercises also benefit patients by minimizing symptoms of depression [7].

Behavioral management algorithms such as the Describe, Investigate, Create, and Evaluate (DICE) approach [34], art, music and dance therapies, recreational therapies such as craft making, games and pet assisted therapies, stimulation-oriented approaches [12] have been used in the AD management.

Miscellaneous non-pharmacological methods which have also been recommended include, memory training with help of computers and, control of sleep disorders by special lighting [1]. These methods improve cognition and performance in activities of daily living. Further these methods reduce behavioural symptoms like aggression, agitation, depression and sleep disturbances [1]. Use of therapeutic touch in the form of massage may decrease agitation or irritability [35]. Sauna bathing was found to be protective against $\mathrm{AD}$ and dementia among middle-aged Finnish men [36]. Active participation in social activities, day programs, and in spiritual activities are also suggested [37]. In light of the limited benefits seen with currently available medications, non-pharmacological methods need to be encouraged more for AD management at this setting.

\section{Limitations}

Information available in few medical records were incomplete. Further research using other 
study designs and data collection methods is therefore warranted at this settings.

\section{CONCLUSIONS}

The age of onset of AD in about one-fourth of patients in the present study was 76 to 80 years. About two-third of patients each were males and were from urban areas. History of depression and of TBI was present among $9.1 \%$ and $7.3 \%$ patients respectively. History of consumption of alcohol and smoking was present in $16.4 \%$ and $12.7 \%$ patients respectively. Clinical features such as disori-

\section{REFERENCES}

1. Alzheimer's Association. 2017 Alzheimer's disease facts and figures. Chicago: Alzheimer's Association; 2017

2. GBD 2016 Dementia Collaborators. Global, regional, and national burden of Alzheimer's disease and other dementias, 1990-2016: a systematic analysis for the Global Burden of Disease Study 2016. Lancet Neurol. 2019;18:88-106.

3. Hebert LE, Weuve J, Scherr PA, et al. Alzheimer disease in the United States (2010-2050) estimated using the 2010 Census. Neurology 2013;80:1778-1783.

4. Schrijvers EM, Verhaaren BF, Koudstaal PJ, et al. Is dementia incidence declining? Trends in dementia incidence since 1990 in the Rotterdam Study. Neurology 2012;78:1456-1463.

5. Satizabal CL, Beiser AS, Chouraki V, et al. Incidence of dementia over three decades in the Framingham Heart Study. N Engl J Med. 2016;374:523-532.

6. Prince MJ, Wimo A, Guerchet M, et al. World Alzheimer Report 2015. The Global Impact of Dementia: An Analysis of Prevalence, Incidence, Cost and Trends. London: Alzheimer's Disease International; 2015.

7. Burns A, lliffe S. Alzheimer's disease. BMJ. 2009;338:b158.

8. Daviglus ML, Plassman BL, Pirzada A, et al. Risk Factors and Preventive Interventions for Alzheimer Disease: State of the Science. Arch Neurol 2011;68:1185-1190.

9. Mathuranath PS, George A, Ranjith N, et al. Incidence of Alzheimer's disease in India: a 10 years follow-up study. Neurol India. 2012;60:625-630.

10. Ganguli M, Chandra V, Kamboh MI, et al. Apolipoprotein E polymorphism and Alzheimer disease:The Indo-US Cross-National Dementia Study. Arch Neurol. 2000;57:824-830.

11. National Institute on Ageing. Alzheimer's Disease Genetics Fact Sheet. Available from: https://www.nia.nih.gov/health/alzheimers-diseasegenetics-fact-sheet [Last Updated: 30 Aug 2015; Accessed 1 Oct 2019].

12. Grossberg GT, Desai AK. Management of Alzheimer's disease. J Gerontol A Biol Sci Med Sci. 2003;58:331-353.

13. Sherrington $R$, Rogaev El, Liang $Y$, et al. Cloning of a gene bearing missense mutations in early-onset familial Alzheimer's disease. Nature. 1995;375:754-760.

14. Huang CC, Chung CM, Leu HB, et al. Diabetes Mellitus and the Risk of Alzheimer's Disease: A Nationwide Population-Based Study. PLoS ONE 2014;9:e87095.

15. Abner EL, Jicha GA, Christian WJ, et al. Rural-Urban Differences in Alzheimer's Disease and Related Disorders Diagnostic Prevalence in Kentucky and West Virginia. J Rural Health. 2016;32:314-320.

16. Kivipelto M, Ngandu T, Laatikainen T, et al. Risk score for the prediction of dementia risk in 20 years among middle aged people: a longitudinal, population-based study. Lancet Neurol. 2006;5:735-741. entation and recent memory loss was present among $70.9 \%$ and $69.1 \%$ patients respectively. Donepezil was prescribed for management in $80 \%$ of AD patients. Non-pharmacological methods like physiotherapy were recommended in $38.2 \%$ AD patients. Considering the varied problems faced by the Alzheimer's patients in this settings, a multidisciplinary approach is advised for the AD management. This will ensure comprehensive assessment and varied interventions to manage the heterogeneity and complexity of AD. Non-pharmacologic methods of the AD management need to be introduced more at this settings.

Conflict of interest: none declared Financial support: none declared

17. Alzheimer Society Canada. Risk factors. Available from: https:// alzheimer.ca/en/Home/About-dementia/Alzheimer-s-disease/ Risk-factors [Last Updated: 24 Aug 2018; Accessed 15 Feb 2019].

18. Brown WR, Thore CR. Review: cerebral microvascular pathology in ageing and neurodegeneration. Neuropathol Appl Neurobiol. 2011;37:56-74.

19. de la Torre JC. Cerebral hemodynamics and vascular risk factors: setting the stage for Alzheimer's disease. J Alzheimers Dis. 2012;32:553-567.

20. Burke SL, Maramaldi P, Cadet T, et al. Associations between depression, sleep disturbance, and apolipoprotein $\mathrm{E}$ in the development of Alzheimer's disease: dementia. Int Psychogeriatr. 2016;28:1409-1424.

21. Leroil LC. Neuro psychiatric aspects of dementia. In: Burns A, O'Brien J, Ames D, editors. Dementia, 3rd Ed. London: Hodder Arnold; 2005. p. 55-64.

22. Graham DI, Gentleman SM, Nicoll JA, et al. Altered beta-APP metabolism after head injury and its relationship to the aetiology of Alzheimer's disease. Acta Neurochir Suppl. 1996;66:96-102.

23. Franzblau M, Gonzales-Portillo C, Gonzales-Portillo GS, et al. Vascular damage: a persisting pathology common to Alzheimer's disease and traumatic brain injury. Med Hypotheses. 2013; 81:842-845.

24. Brunnstrom HR, Englund EM. Cause of death in patients with dementia disorders. Eur J Neurol 2009;16:488-492.

25. Leon J, Cheng CK, Neumann PJ. Alzheimer's disease care: costs and potential savings. Health Aff (Millwood). 1998;17:206-216.

26. Burns A, O'Brien J. Clinical practice with anti-dementia drugs: a consensus statement from British Association for Psychopharmacology. J Psychopharmacol. 2006;20:732-755.

27. Neugroschl J, Wang S. Alzheimer's disease: diagnosis and treatment across the spectrum of disease severity. Mt Sinai J Med. 2011;78:596-612.

28. Holmes. The efficacy of donepezil in the treatment of neuropsychiatric symptoms in Alzheimer disease. Neurology 2004;63:214-219.

29. Nelson J, Chouinard G. Guidelines for the clinical use of benzodiazepines: pharmacokinetics, dependency, rebound and withdrawal. Can J Clin Pharmacol. 1999;6:69-83.

30. Prince M, Albanese E, Guerchet M, et al. Nutrition and dementia. A review of available research. London: Alzheimer's Disease International; 2014.

31. Barnes S, Wasielewska A, Raiswell C, et al. Exploring the mealtime experience in residential care settings for older people: an observational study. Health Soc Care Community 2013;21:442-450. 
32. Phillips LR, Van OS. Measurement of mealtime interactions among persons with dementing disorders. J Nurs Meas. 1993;1:41-55.

33. Farina N, Rusted J, Tabet N. The effect of exercise interventions on cognitive outcome in Alzheimer's disease: A systematic review. Int Psychogeriatr. 2014;26:9-18.

34. Ellison JM. Clinical Trials for Alzheimer's Disease: What's New? Available from: https://www.brightfocus.org/alzheimers/article/ clinical-trials-alzheimers-disease-whats-new [Accessed 19 February 2019].
35. Snyder M, Egan EC, Bruns KR. Efficacy of hand massage in decreasing agitation behaviors associated with care activities in persons with dementia. Geriatr Nurs. 1995;16:60-63.

36. Laukkanen T, Kunutsor S, Kauhanen J, et al. Sauna bathing is inversely associated with dementia and Alzheimer's disease in middle-aged Finnish men. Age Ageing. 2017;46:245-249.

37. Long CO. Palliative care for advanced dementia. J Gerontol Nurs. 2009;35:19-24. 